\title{
Partizipation in der Frühpädagogik - die ICF als disziplinverbindendes Element
}

\author{
Britta Gebhard · Carina Völlm • Astrid Fink
}

Eingegangen: 30. Oktober 2020 / Angenommen: 1. April 2021 / Online publiziert: 9. Juli 2021

(C) Der/die Autor(en) 2021

Zusammenfassung Teilhabe und Partizipation werden in verschiedenen Fachdisziplinen thematisiert, wobei eine einheitliche Definition und Nutzung der Begriffe fehlen. Im Zuge der Neugestaltung der Eingliederungshilfe werden u. a. sozialgesetzliche Perspektiven auf Teilhabe und Partizipation, die eher den Rehabilitationsund Versorgungswissenschaften zuzuordnen sind, relevant für die inklusive Frühpädagogik. Diese werden in Verbindung mit der Internationalen Klassifikation der Funktionsfähigkeit, Behinderung und Gesundheit (ICF) vorgestellt und es werden Bezüge zu frühpädagogisch bedeutsamen Theoriediskursen und Forschungsständen hergestellt. Möglichkeiten der interdisziplinären Zusammenarbeit zur Teilhabeförderung in der inklusiven frühkindlichen Bildung und Frühförderung werden anhand der ICF aufgezeigt.

Schlüsselwörter Teilhabe $\cdot$ Partizipation $\cdot$ Frühpädagogik $\cdot$ Frühförderung $\cdot$ ICF

Prof. Dr. Britta Gebhard $(\bowtie) \cdot$ Carina Völlm

Bildungs- und Gesellschaftswissenschaften, FH Südwestfalen Standort Soest, Soest, Deutschland

E-Mail: gebhard.britta@fh-swf.de

Carina Völlm

E-Mail: voellm.carina@fh-swf.de

Dr. Astrid Fink

Institut für Medizinische Soziologie, Martin-Luther-Universität Halle-Wittenberg, Halle (Saale), Deutschland

E-Mail: astrid.fink@medizin.uni-halle.de 


\title{
Participation in early childhood education - the ICF as an element connecting disciplines
}

\begin{abstract}
Participation is currently being discussed in different disciplines, although there is no consistent definition or utilization of the term. In German language, participation can be translated into two different terms: "Partizipation" (='participation') and "Teilhabe" (='take part'). In context of the redesign of the German law for integrational assistance, social law perspectives on participation are relevant for inclusive early childhood education, even though they are more likely to be associated with rehabilitation and healthcare sciences. Social law perspectives are presented in connection with the International Classification of Functioning, Disability and Health (ICF) and references are made to theoretical discourses and state of research which are relevant to early childhood education. Possibilities of interdisciplinary cooperation on promoting participation in inclusive early childhood education and early childhood intervention are shown using the ICF.
\end{abstract}

Keywords Participation · Early childhood education · Early childhood intervention $\cdot$ ICF

Die Begriffe Partizipation und Teilhabe werden derzeit sehr vielfältig genutzt, dabei jedoch z. T. synonym verwendet oder klar voneinander abgegrenzt. Im frühpädagogischen Kontext werden sie z. B. als Ziel der Arbeit, als Konzeptbestandteil oder als Methode beschrieben und in der Praxis umgesetzt (s. unten Bildungspläne, Sozialgesetzgebung, Publikationen, Konzeptionen). Es entsteht jedoch der Eindruck, dass Partizipation und Teilhabe dabei unzureichend definiert werden und somit unklar ist, was explizit gemeint ist. Dies hat bereits zu einer grundlegenden Aufarbeitung in verschiedenen frühpädagogisch relevanten Fachpublikationen geführt (u.a. Hansen et al. 2011; Prengel 2016; Spreer et al. 2019; Weiß 2019).

Im Zuge der fortschreitenden inklusiven frühpädagogischen Bildung, Erziehung und Betreuung aller Kinder wird es zunehmend zum Selbstverständnis, dass Kindern mit (drohenden) Beeinträchtigungen allgemeine frühpädagogische Einrichtungen offenstehen (u. a. Bertelsmann Stiftung 2019 in Bezug auf Kinder, die Eingliederungshilfeleistungen beziehen). Um ein entwicklungsförderliches, auf den individuellen Bedarf ausgerichtetes Bildungs-, Erziehungs- und Betreuungsangebot zu schaffen, wird die interdisziplinäre Zusammenarbeit mit der Frühförderung sowie weiteren Förder-, Therapie- und Unterstützungsangeboten zu einem selbstverständlichen Prozess innerhalb frühpädagogischer Bildungseinrichtungen. Frühförderstellen werden, neben dem Jugendamt sowie niedergelassenen therapeutischen Einrichtungen, als einer der meistgenannten Kooperationspartner von Kindertageseinrichtungen aufgeführt (Wiedebusch et al. 2015; Peucker et al. 2017; Heimlich und Ueffing 2018).

Aus der Frühförderperspektive geht es darum, Partizipation als Endpunkt, d.h. Ziel einer Versorgung bzw. Förderung zu begreifen (Urschitz et al. 2016). Gesetzliche Grundlage der Frühförderung bildet das Sozialgesetzbuch (SGB) IX ,Rehabilitation und Teilhabe von Menschen mit Behinderungen“. Frühförderung wird hier als eine Leistung neben Weiteren zur Sicherung der sozialen Teilhabe angesehen 
(§ 76 SGB IX), um eine gleichberechtigte Teilhabe am Leben in der Gemeinschaft zu ermöglichen oder zu erleichtern. In frühkindlichen Bildungseinrichtungen wird Partizipation eher als Prozess verstanden bzw. als Gestaltungsprinzip (u. a. Hansen et al. 2011) oder als soziale Teilhabe im Sinne eines ,Mitmachen Dürfens', d.h. Teilnehmens (Weiß 2019). Bei kooperativ miteinander arbeitenden Fachdisziplinen kann diese Diskrepanz zu Missverständnissen führen. Eine Basis für eine ,gemeinsame Sprache' (Simon und Philippi 2019) bzgl. Partizipation und Teilhabe kann hierbei die Internationale Klassifikation der Funktionsfähigkeit, Behinderung und Gesundheit (ICF) der Weltgesundheitsorganisation (WHO) bieten.

Um die ICF als disziplinverbindendes Elemente im Kontext von Partizipation in der frühkindlichen Bildung zu fokussieren, wird im Folgenden eine allgemeine Einleitung zu Partizipation und Teilhabe vorgenommen. Auf dieser Basis wird die ICF und ihre Implementierung in die Sozialgesetzgebung erläutert. Die Notwendigkeit, sich mit der ICF konzeptionell-inhaltlich auseinanderzusetzen, wird hergeleitet und beschrieben, da diese im Zuge der Beteiligung am Bedarfsermittlungsverfahren zur Gewährung von Eingliederungshilfe als aktuelle Thematik im Kontext der inklusiven Frühpädagogik, gerade auch bezogen auf eine interdisziplinäre Zusammenarbeit, derzeit noch wenig thematisiert wird. Anschließend werden ausgewählte, für eine inklusive frühpädagogische Bildung relevante und über das Begriffsverständnis der Partizipation, wie es in der ICF angeführt wird, hinausgehende Theorien und Perspektiven von Partizipation und Teilhabe beleuchtet, um Schnittmengen von Frühpädagogik und Frühförderung aufzuzeigen. Chancen, die in diesem neu eingeführten Prozess der Eingliederungshilfe zur Gestaltung von gelingenden, interdisziplinären Förder-, Bildungs- und Erziehungsangebote für Kinder mit (drohenden) Beeinträchtigungen liegen, werden anhand aktueller empirischer Ergebnisse abschließend aufgegriffen und diskutiert. Es muss betont werden, dass sich die folgenden Ausführungen im Schwerpunkt auf die inklusive Bildung, Erziehung und Betreuung von Kindern mit und ohne (drohenden) Beeinträchtigungen beziehen. Dies soll weder einem aktuellen Inklusionsverständnis unter Beachtung verschiedener, miteinander verwobener Heterogenitätsdimensionen widersprechen, noch ein dichotomes Verständnis von Behinderung vs. Nicht-Behinderung ausdrücken. Diese Eingrenzung wird vor dem Hintergrund der Fokussierung auf das SGB IX (Eingliederungshilfe) vorgenommen, die dem Personenkreis der Menschen mit (drohenden) Beeinträchtigungen vorbehalten ist.

\section{Partizipation und Teilhabe - eine begriffliche Differenzierung und Annäherung}

Weiß (2019) greift die begriffliche Verwendung von Partizipation und Teilhabe auf und weist darauf hin, dass der international gebräuchliche Begriff "participation“ (Partizipation), wie er u.a. in der UN-Behindertenrechtskonvention (UN-BRK) genutzt wird, in der deutschen Übersetzung sowie in der aktuellen Sozial-, Bildungsund Gesundheitspolitik und Gesetzgebung fast durchgängig mit „Teilhabe“ übersetzt wird; hier wird also scheinbar von einer Synonymität ausgegangen. Die deutsche Übersetzung der UN-BRK in „Teilnahme“ und „Teilhabe“, also eine dritte Differen- 
zierung, unterscheidet sich jedoch laut Hirschberg (2010) von „Partizipation“, und es gehe hierdurch der Mitbestimmungsaspekt verloren. In der ICF wird hingegen durchgängig die Doppelbegrifflichkeit Partizipation [Teilhabe] verwendet.

Die Partizipation und Teilhabe junger Kinder und ihrer Familien zu sichern sowie zu fördern ist in den Bildungsplänen der verschiedenen Bundesländer verankert. In diesen ist die demokratische Partizipation bzw. Gestaltung von Teilhabe als Zielsetzung formuliert. So heißt es in den Bildungsgrundsätzen NRW (MSW 2018, S. 13): „Gesellschaftliche Teilhabe ist das Leitziel einer Bildung von Anfang an. Sie zielt auf die Schaffung von ungehinderten Zugängen zu gesellschaftlichen Ressourcen und die Eröffnung und Entwicklung von gleichen Chancen für alle“. Zudem soll Kindern Teilhabe an Entscheidungsprozessen (Partizipation) ermöglicht werden (ebd.). In den Bildungsgrundsätzen werden die Begrifflichkeiten Partizipation und Teilhabe nahezu synonym eingesetzt, Partizipation jedoch eher in einer demokratiebezogenen Perspektive bezogen auf Entscheidungsprozesse. Bereits diese Ausführungen zeigen, wie divers die begriffliche Verwendung vorgenommen wird.

In der UN-BRK wird Teilhabe als Querschnittsanliegen bezeichnet. Dabei ist oberstes Ziel, den vollen und gleichberechtigten Genuss aller Menschenrechte und Grundfreiheiten zu fördern sowie die volle, wirksame und gleichberechtigte Teilhabe von Menschen mit Behinderungen an der Gesellschaft zu erreichen (Art. 1 UN-BRK). Teilhabe als Grundsatz wird in Art. 3 in Bezug auf ein inklusives Bildungssystem dahingehend aufgegriffen, dass Menschen mit Behinderungen und ihre Organisationen beim Aufbau eines inklusiven Bildungssystems einbezogen werden müssen (Partizipation) und dass das Recht auf Zugang gewährt werden muss. Aufgabe in frühkindlichen Bildungsangeboten ist es, hierfür eine Grundlage zu schaffen, damit Kinder lernen und spüren, dass ihre Bedürfnisse und Interessen wahrgenommen werden, was sich z.B. darin zeigen kann, dass sie beteiligt werden (Prengel 2016).

Thon und Mai (2017) bezeichnen Teilhabe in Bezug auf Frühpädagogik als einen Begriff an der Schnittstelle zwischen Sozialpolitik und Bildungspolitik, denn durch Bildung soll gesellschaftliche Teilhabe gewährleistet werden, jedoch wird politisch auch gefordert, dass Teilhabe an Bildung ermöglicht werden soll (Teilhabe an und durch Bildung). Kritisch gehen sie auf die Pädagogisierung dieses Anliegens ein, das in ihrer Lesart folgendermaßen verstanden wird: „So lässt sich auch gesellschaftliche ,Teilhabe' als etwas konzipieren, das durch die Schaffung bestimmter Voraussetzungen im einzelnen Subjekt - im vorliegenden Fall im frühpädagogisch zu fördernden Kind - gewährleistet werden muss. Dieses Subjekt gilt es mit entsprechendem Wissen und Kompetenzen auszustatten“ (Thon und Mai 2017, S. 262). Diese Sichtweise lässt die Aspekte der Anerkennung (Zugehörigkeitsgefühl) und der mitgestaltenden Teilhabe eher außer Acht (Weiß 2019).

Partizipation im Kontext von Bildung und Förderung wird von Weiß (2019) in Bezug auf das Verhältnis von Partizipation und Bildung betrachtet: Geht es nur um eine reine Teilnahme, im Sinne von Mitmachen dürfen, wären ,Bildung und Partizipation“ oder ,Förderung und Partizipation“ additive Begriffspaare. Partizipation sollte jedoch als mehr begriffen werden als nur dabei zu sein (Teilnahme - Attendance), denn es geht um das Mitwirken und ein aktives Beteiligt-Sein (Involvement), um mitgestaltend Einfluss auf die Lebensumstände nehmen zu können. Bedeutend 
ist hier auch das subjektive Zugehörigkeitsgefühl. Dem Kind kommt folglich eine mitgestaltende Rolle in Bildungs- und Förderprozessen zu, so dass nicht von einer additiven Verknüpfung von Bildung und Partizipation oder Förderung und Partizipation gesprochen werden kann, sondern vielmehr sollte von einem Doppelcharakter ausgegangen werden: Kinder haben ein Recht auf Teilhabe, z. B. an Bildung, Förderung oder Therapie; diese sollen gleichzeitig aber zu dem Zwecke eingesetzt werden, Teilhabemöglichkeiten zu erhöhen. Hierdurch sind Bildung und Förderung einerseits „Vorbereitungsinstanzen“ für (spätere) Teilhabe, gleichzeitig nehmen sie aber mitgestaltend Einfluss auf die Prozesse der Bildung und Förderung, d.h. sie sollen in die Planung von Lern-, Bildungs-, Förder- und Erziehungsprozesse einbezogen werden (ebd.). Dies wirft auch Fragen an eine „Partizipationsfähigkeit“ aus entwicklungspsychologischer Perspektive auf, welche hier jedoch nicht weiter aufgegriffen werden können, sondern nur in den Ausführungen zum bereits ab Geburt existierenden Recht auf Partizipation (u. a. Hansen et al. 2011) angerissen werden.

In diesem Fachartikel werden Partizipation und Teilhabe in gemeinsamer Nennung genutzt mit leicht unterschiedlicher Konnotation. Hierdurch wird versucht der Komplexität der Begrifflichkeiten gerecht zu werden. Teilhabe steht dabei vermehrt für den Zugang und die Möglichkeit sowie das subjektive Erleben; Partizipation fokussiert stärker die Mitbestimmung und Mitgestaltung von Teilhabeprozessen.

\section{Sozialgesetzliche Perspektive: Bundesteilhabegesetz, Eingliederungshilfe und ICF}

Durch die schrittweise Einführung des Bundesteilhabegesetztes (BTHG) sollen die Vorgaben, die sich aus der UN-BRK u. a. für eine inklusive Bildung ( $\$ 24$ ) ableiten lassen, umgesetzt werden. Damit verbunden ist ein Paradigmenwechsel: weg von der Fürsorgepflicht, hin zum Teilhaberecht. Das Ziel ist, Menschen mit Behinderungen eine gleichberechtigte, vollständige und vor allem gelingende, d.h. wirksame Teilhabe an verschiedenen Lebensbereichen zu schaffen (BMAS 2016). Diese Lebensbereiche umfassen politisches, gesellschaftliches, wirtschaftliches und kulturelles Leben. Mit dem Wechsel zum Teilhaberecht wird Abstand genommen von der Ausgrenzung, der Sorge um oder für jemanden, ohne dass die Person, bzw. der Mensch mit Behinderung selbst mitbestimmen konnte und beteiligt wurde, also partizipieren konnte. Gesellschaftlich gilt weiterhin die Werthaltung des Füreinander-Sorgens und Sich-Kümmerns, nun steht jedoch die Selbstbestimmung im Fokus: Fürsorge darf nicht entmündigen. Die Äußerung des eigenen Willens muss durch ein Wahl- oder Wunschrecht realisiert werden (ebd.).

In das SGB IX wurde die ICF aufgenommen, eine Klassifikation mit welcher ergänzend zu einer klassischen medizinischen Diagnose die Funktionsfähigkeit, Behinderung und Gesundheit von Menschen beschrieben werden kann. Diese basiert auf einem bio-psycho-sozialem Erklärungsansatz mit Wechselwirkungen zwischen verschiedenen Komponenten (Abb. 1). Anhand dieses Modells kann definiert werden, was eine Behinderung ist und wann eine solche überhaupt erst entsteht. 
Abb. 1 Wechselwirkungen zwischen den Komponenten der ICF (WHO 2005, S. 21)

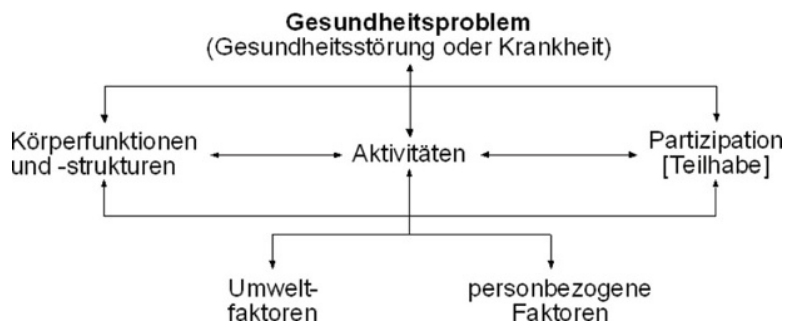

Die ICF liegt aktuell (noch) in einer Version für Erwachsene (WHO 2005) und einer Version für Kinder und Jugendliche, der ICF-CY (WHO 2011) vor; eine Vereinigung der Versionen ist geplant.

In $\S 2$ Abs. 1 SGB IX wurde durch dieses Modell die Wechselwirkung eines Gesundheitsproblems mit einstellungs- und umweltbedingten Barrieren, d.h. Kontextfaktoren, als neue Sicht auf Behinderung aufgenommen. Das entspricht dem Verständnis der UN-BRK, welche auf der ICF basiert und Behinderung als Teilhabeeinschränkung darstellt. Folglich wird Behinderung im SGB IX nicht mehr als Eigenschaft und Defizit einer Person betrachtet, sondern als gesundheitliche Beeinträchtigung im Zusammenspiel mit Kontextfaktoren sowie den Interessen und Wünschen des betroffenen Menschen. Die bio-psycho-soziale Sichtweise sagt aus, dass das Vorhandensein einer Krankheit oder einer Schädigung bzw. Funktionsbeeinträchtigung an sich keine Behinderung darstellt. Diese entstehen erst in Wechselwirkung mit anderen Komponenten - personbezogenen und umweltbezogenen Faktoren -, die sich als gesamter Kontext auf die Aktivität und Partizipation [Teilhabe] einer Person auswirken und somit in einer Teilhabeeinschränkung münden könnte. In der ICF wird „Behinderung“ als mehrdimensionales Phänomen verstanden, welches weniger die Person als vielmehr die Situation, in der sich eine Person befindet, beschreibt. Behinderung ist folglich situationsabhängig (Hollenweger 2015).

Durch das BTHG erfahren auch die Leistungen der Eingliederungshilfe eine inhaltliche Neuausrichtung. Sie werden mit dem Ziel erbracht, Behinderung oder deren Folgen zu beseitigen oder zu mildern und dadurch eine gleichberechtigte Teilhabe am gesellschaftlichen Leben zu ermöglichen. Diese Leistungen haben Partizipation und Teilhabe in den verschiedenen Lebensbereichen als Endpunkt bzw. Ziel einer Versorgung. Eine weitere wesentliche Veränderung in der Eingliederungshilfe im Jahr 2020 stellt die Einführung des Teilhabe- und Gesamtplanverfahrens (sog. Bedarfsermittlungsverfahren) dar. Hierdurch erhalten die betroffenen Personen mehr Mitbestimmung, was der Abwendung vom Fürsorgerecht entspricht. Zudem sollen die Leistungen, die verschiedene Einrichtungen und Institutionen erbringen, z. B. Kita und Frühförderung, besser aufeinander abgestimmt und miteinander besprochen werden ( $\$ 20$ SGB IX Teilhabeplankonferenz). In § 118 SGB IX wird vorgegeben, dass sich die Instrumente der Bedarfsermittlung an der ICF zu orientieren haben. Die notwendige Unterstützung soll sich dadurch künftig konsequenter an den individuellen Bedarfen und Wünschen der Menschen mit Behinderung orientieren.

Dieses eher einer gesundheits- und rehabilitationswissenschaftlichen Fachperspektive zuzuordnende Grundverständnis von Partizipation und Teilhabe scheint 
aktuell in der Frühpädagogik weniger bekannt zu sein. Der ICF scheint im Bildungsbereich eher mit Skepsis begegnet zu werden aufgrund der Herkunft aus dem Gesundheitsbereich (Hollenweger 2015). Die ICF bietet der Frühpädagogik jedoch im Hinblick auf Partizipation und Teilhabe wertvolle Impulse und Überschneidungen mit bereits etablierten Theorieansätzen, die nachstehend aufgezeigt werden.

\section{Beschreibung und Möglichkeiten der ICF}

„Allgemeines Ziel der ICF ist, einen Rahmen zur Beschreibung von Gesundheitsund mit Gesundheit zusammenhängenden Zuständen zur Verfügung zu stellen“ (WHO 2011, S. 35). Wichtig ist, dass manche Umstände, wie z. B. sozialer Hintergrund, ethnische Zugehörigkeit, Geschlecht oder Religion hier nicht miterfasst werden, da sie sich in der Regel nicht unmittelbar auf einen Gesundheitszustand, der zu Beeinträchtigung der Partizipation führen kann, auswirken (ebd., S. 39). Diese Faktoren haben jedoch eine hohe Relevanz und können im Bereich der personbezogenen Faktoren aufgenommen werden, die nicht einheitlich klassifiziert, sondern frei beschreibbar sind. Die ICF soll als gemeinsame und verständliche Sprache für Fachkräfte und Betroffene dienen. Die einzelnen Komponenten des bio-psycho-sozialen Modells (s. Abb. 1), bilden die Struktur der ICF.

In der ICF wird Partizipation [Teilhabe] als „Einbezogensein in eine Lebenssituation“ und als die ,gesellschaftliche Perspektive der Funktionsfähigkeit“ definiert (WHO 2011, S. 46). An dieser recht knappen Definition zeigt sich bereits, dass es sich nicht um individuelle Eigenschaften handelt, sondern um etwas, das mit der Gesellschaft in Zusammenhang steht. Die ICF geht davon aus, dass ein Gesundheitsproblem, verstanden als eine Einschränkung in den Aspekten des Lebens und der individuellen Lebensführung, durch die verschiedenen Komponenten beeinflusst wird, die in Wechselwirkung zueinanderstehen (Abb. 1). Referenzpunkt ist immer eine Person ohne Gesundheitsproblem oder Behinderung der entsprechenden Kultur oder Gesellschaft. Diese Hinzufügung ist vor allem für den Bereich der Partizipation [Teilhabe] wichtig, da dieser häufig durch kulturell oder sozialisationsgeprägte Interessen und Bedürfnisse bestimmt wird. Als veranschaulichendes und stark vereinfachtes Beispiel für die Wechselwirkung der verschiedenen Komponenten kann ein Kind mit einer angeborenen Querschnittslähmung aufgrund einer Spina bifida, d.h. eines „offenen Rückens“ dienen. Die Körperstruktur von Nervensystem und Muskulatur sind geschädigt, was die Körperfunktionen von Kraftaufbau in den Beinen oder Bewegung von Gelenken wie z. B. den Knien und Hüftgelenken einschränkt. Daher kann das Kind die Aktivität „frei gehen“ nicht ausführen und ist mit einem Rollstuhl versorgt. Sind die Umweltfaktoren im Bereich „Einstellung von Fachkräften“" in der Kita nun bspw. so, dass die pädagogischen Fachkräfte sich nicht in der Lage sehen, ein nichtgehendes Kind zum Waldausflug mitzunehmen oder den Ausflug so zu gestalten, dass ein Kind im Rollstuhl teilnehmen kann, so wird es in seiner gewünschten Partizipation [Teilhabe] „mit den anderen Kindern beim Ausflug dabei sein“ eingeschränkt und „,behindert“. Ein positives Beispiel im Bereich der Umweltfaktoren könnte die Ausstattung einer Kita mit höhenverstellbaren und unterfahrbaren Tischen sein: Hierdurch kann das Kind eigenständig und 
ohne Schwierigkeiten an den Tisch zu den anderen fahren, um gemeinsam zu essen. Damit ist es in seiner Partizipation [Teilhabe] bzgl. „Gemeinsam essen in der Kita“ nicht eingeschränkt. Auch wenn eine Querschnittslähmung ein gravierendes Gesundheitsproblem darstellt, soll dieses Beispiel verdeutlichen, dass erst durch die Wechselwirkung z. B. mit fördernden oder hemmenden Umweltfaktoren eine Teilhabeeinschränkung und damit eine „Behinderung“ entsteht. Wechselwirkungen können auf der Umweltebene durch materielle (z.B. bauliche), soziale und einstellungsbezogene Faktoren (z.B. Einstellung der Fachkräfte zu Inklusion, s. oben) bestehen. Wechselwirkungen können aber auch mit den personbezogenen Eigenschaften, wie dem Charakter oder den Interessen des Kindes, zusammenhängen.

Der Bereich der Aktivitäten und Partizipation [Teilhabe] ist der Kern und eine Besonderheit der ICF: Er bezieht sich auf insgesamt neun Lebensbereiche (life domains), die auch im SGB IX/BTHG aufgegriffen werden, um die Partizipation [Teilhabe] einer Person im Rahmen der Bedarfsermittlungsinstrumente gut einschätzen zu können. Für diese müssen Beeinträchtigungen der Aktivität und Partizipation [Teilhabe], welche nicht nur vorübergehend sind, in den folgenden Lebensbereichen der ICF beschrieben werden: 1 - Lernen und Wissensanwendung, 2 - Allgemeine Aufgaben und Anforderungen, 3 - Kommunikation, 4 - Mobilität, 5 - Selbstversorgung, 6 - Häusliches Leben, 7 - Interpersonelle Interaktionen und Beziehungen, 8 - Bedeutende Lebensbereiche, 9 - Gemeinschafts-, soziales und staatsbürgerliches Leben. Hierbei besteht der Anspruch, durch diese neun Lebensbereiche sämtliche Aktivitäten und Bereiche der Partizipation [Teilhabe], welche Menschen nutzen, abzubilden.

\section{Partizipation und Teilhabe - eine multiperspektivische Betrachtung verschiedener theoretischer Fundierungen im Kontext von Frühpädagogik und Frühförderung}

Von Kardorff (2014) führt vier Diskussionslinien über Partizipation auf: a) die aktive Teilnahme an Entscheidungsprozessen; b) Anspruch auf gleichberechtigte/inklusive Teilhabe u.a. an Bildung; c) diskriminierungsfreie Anerkennung von Verschiedenheit und soziale Einbindung in die Gesellschaft (Teil-Sein); d) Teil-Gabe als gesellschaftliche Reziprozitätserwartung für gewährte Leistungen durch die aktive Übernahme von Selbstverantwortung und sozial-moralischen Verpflichtungen. Vor allem a) bis c) finden sich inhaltlich an verschiedenen Stellen im Folgenden wieder.

Ausgehend vom konzeptionellen Verständnis von Partizipation [Teilhabe] der ICF als „Eingebundensein in eine Lebenssituation“ (WHO 2005, S. 19) erfolgt in einer Fußnote (Nr. 14, S. 20) eine wichtige inhaltliche Ergänzung: Die Definition von Partizipation [Teilhabe] führt das Konzept des Einbezogenseins ein, womit Vorstellungen von ,teilnehmen an“, ,teilhaben an“, ,beschäftigt sein in“, (Diskussionslinie b) ,,anerkannt werden“ (Diskussionslinie c) oder „Zugang zu benötigten Ressourcen haben“" (Diskussionslinie a und b) verbunden werden.

Der letzte Aspekt (Zugang zu benötigten Ressourcen haben) lässt sich gut mit einer kinderrechtlichen Perspektive verbinden. Liebel (2013, S. 101) differenziert die Konstrukte Teilhabe und Partizipation aus einer kinderrechtlichen Perspektive: 
Teilhabe definiert er als „die Art und Weise [...] in der Menschen Zugang zu den Prozessen, Institutionen, Gütern und Leistungen in einer bestimmten Gesellschaft haben“. Er bezieht sich dabei auf ein Spannungsfeld zwischen sozialer Inklusion und Exklusion. Herrscht in einer Gesellschaft eine große soziale Ungleichheit, so besteht zwischen den Teilhabechancen einer privilegierten Minderheit und einer benachteiligten Mehrheit eine große Diskrepanz. Die weitere Bedeutung von Partizipation, Teilnahme oder Beteiligung sieht er im Bereich der Äußerung des freien Willens und (Mit-)Entscheidungsmöglichkeiten von Individuen oder sozialen Gruppen, was sich nicht nur in einer Mitgliedschaft, sondern in aktivem Handeln in sämtlichen privaten wie öffentlichen Bereichen auswirkt. Den von Liebel (2013) genannten Exklusionsaspekt als Gegenpol von Teilhabe beschreibt Kronauer (2017, S. 1) als ,,...] die Ausschließung von Menschen von grundlegenden Voraussetzungen der Teilhabe am gesellschaftlichen Leben“.

Besonders der von Liebel aufgegriffene Aspekt der Beteiligung an Entscheidungen wird auch von Knauer (2012, S. 65) aus einer ebenfalls kinderrechtlichen Perspektive weiter differenziert: „Partizipation - das Recht auf Anhörung und Beteiligung ist neben dem Recht auf Schutz, auf Entwicklung und auf Förderung eines der Grundprinzipien der in der UN-Konvention festgeschriebenen Kinderrechte“. Mit diesem demokratischen Verständnis von Kinderrechten sollen Kinder an Planungsund Entscheidungsprozessen in frühkindlichen Bildungseinrichtungen beteiligt werden. Dies stellt ein Grundrecht dar, das nicht an Voraussetzungen, wie z. B. ein bestimmtes Entwicklungsalter, die sprachliche oder kognitive Fähigkeit etc. gebunden ist. Hierbei stehen die pädagogischen Fachkräfte in der Pflicht, Kindern Möglichkeiten zu eröffnen sich auszudrücken und ernstgenommen mitzuwirken durch Impulse für die Qualitätsentwicklung, was einerseits ihr Recht, andererseits ihr Bedürfnis ist. Es zeigt sich in diesem Zusammenhang als eine zentrale pädagogische Herausforderung, dem Recht der Kinder, sich zu bilden und dabei von pädagogischen Fachkräften angeregt, begleitet, inspiriert und gestärkt zu werden, gerecht zu werden, dabei aber ihr Recht auf Spiel, Ruhe und Erholung nicht zu missachten (Nentwig-Gesemann und Maywald 2020).

Beteiligung an Entscheidungen hat in der Philosophie der ICF und in dem BTHG eine bedeutsame Rolle: So wird von der Bundesarbeitsgemeinschaft für Rehabilitation (BAR 2016, S. 11) als elementares Handlungsprinzip gefordert, ,den betroffenen Menschen als aktiven, gleichberechtigen und für sich Eigenverantwortung tragenden Partner an[zu]sehen“. In der ICF wird im Bereich der ethischen Leitlinien beschrieben, dass die ICF immer so eingesetzt werden sollte, dass unter Mitwirken der betroffenen Person ihre Wahl- und Steuerungsmöglichkeiten bzgl. ihres Lebens erhöht werden.

Wright et al. (2010) beschreiben eine adaptierte Anwendung des Stufen-Modells „ladder of citizen participation“ von Arnstein im Kontext der partizipativen Gesundheitsforschung, um die Partizipation von Patientinnen und Patienten kritisch zu reflektieren (Abb. 2). Damit verbunden ist die Annahme, dass die realisierte Partizipation einer Person umso größer ist, je mehr Einfluss sie auf den Entscheidungsprozess hat (Wright et al. 2007). Dieses Modell lässt sich auch auf Frühförderprozesse oder Situationen aus der frühkindlichen Bildung übertragen. 


\begin{tabular}{|c|c|}
\hline Selbstorganisation & $\begin{array}{l}\text { Über Partizipation } \\
\text { hinaus }\end{array}$ \\
\hline Entscheidungsmacht & \multirow{3}{*}{ Partizipation } \\
\hline Teilweise Entscheidungskompetenz & \\
\hline Mitbestimmung & \\
\hline Einbeziehung & \multirow{3}{*}{$\begin{array}{l}\text { Vorstufen der } \\
\text { Partizipation }\end{array}$} \\
\hline Anhörung & \\
\hline Information & \\
\hline Anweisung & \multirow{2}{*}{ NichtPartizipation } \\
\hline nstrumentalisierung & \\
\hline
\end{tabular}

Abb. 2 Stufen der Partizipation in der Gesundheitsförderung (Wright et al. 2010, S. 42) (C) Hogrefe Verlag. Mit freundlicher Genehmigung des Hogrefe Verlages Bern

Anhand der Stufen kann reflektiert werden, zu welchem Grad Kinder und auch deren Familien partizipieren können: Liegt die gesamte Entscheidungskompetenz, aber auch alles Wissen z. B. nur bei der Einrichtung/den Fachkräften, so findet keine Partizipation statt. Werden Kinder und/oder ihre Familien nach ihren Interessen befragt, über den aktuellen Prozess informiert und einbezogen, findet eine Vorstufe der Partizipation statt. Von ,wirklicher“ Partizipation kann erst gesprochen werden, wenn mitbestimmt und (teilweise) entschieden werden kann sowie eine Teilung der Entscheidungsmacht stattfindet.

Hierin liegt z. T. auch die Kritik begründet, dass einzelne Programme, die in Kindertageseinrichtungen zur Partizipation durchgeführt werden, meist auf Ebene der Anhörung verweilen, aber eine Entscheidungskompetenz und Entscheidungsmacht häufig nicht erreichen (Prengel 2016). Bezogen auf die Frühförderung bedeutet Partizipation vor allem, dass Ziele von den Eltern und Kindern formuliert werden, um gemeinsam mit den Fachpersonen einen Handlungsplan aufzustellen (Pretis 2016). Damit ändert sich die Rolle der Fachkräfte hin zu einer mehr beratenden Funktion.

Teilnehmen und teilhaben wurden bereits in Bezug auf Weiß (2019) ausgeführt und stellen eine für die inklusive Frühpädagogik bereits vielfach rezipierte fachliche Forderung dar: „Dabeisein ist nicht alles“ (Kreuzer und Ytterhus 2013). Dabei wird die Gestaltung von Teilhabeprozessen in der Praxis immer wieder kritisch angemahnt (u.a. Sarimski 2015). Die subjektive Perspektive des Einbezogenseins (,Zugehörigkeitsgefühl“) (WHO 2005) ist wichtig und von einer Außenperspektive (Teilnahme, Attendance) zu unterscheiden. Imms et al. $(2016,2017)$ haben ein multidimensionales Konzept des komplexen Konstrukts Partizipation erstellt. Datenbasis waren wissenschaftliche Arbeiten zu Kindern mit Behinderungen. Sie analysierten verschiedene relevante auf die Partizipation und Teilhabe einflussnehmende Aspekte, aus denen sie das theoretische Konstrukt „family of participation related constructs“ erstellten (s. Abb. 3). 


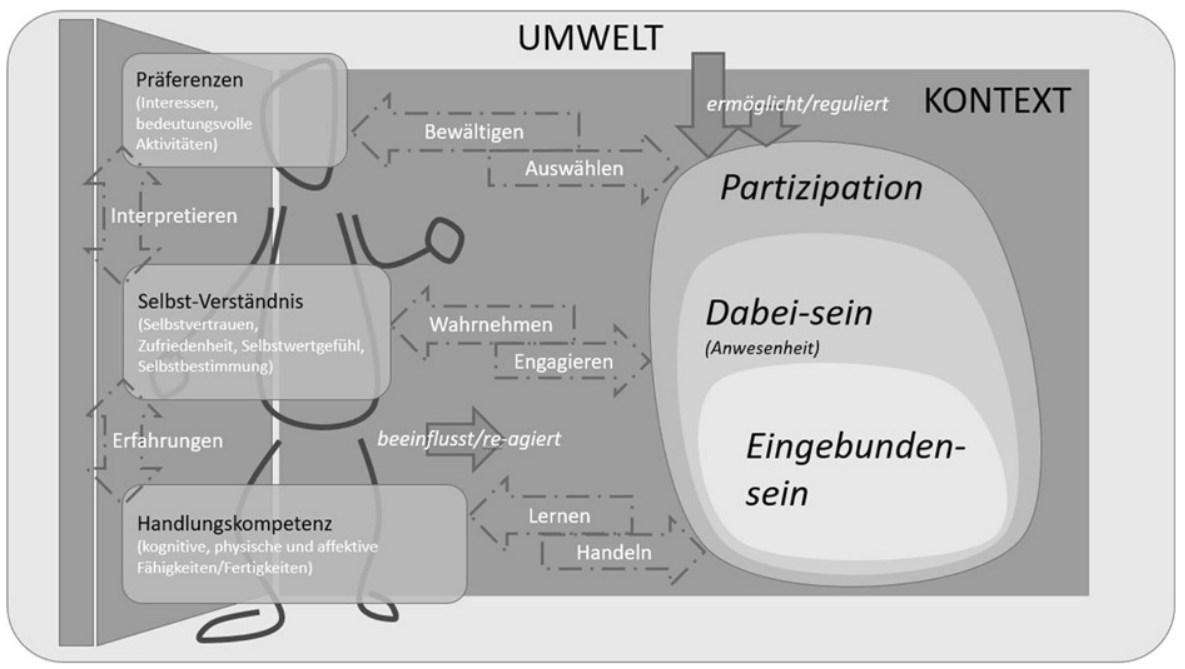

Abb. 3 Die Familie der partizipationszugehörigen Konstrukte mit person- und umweltzentrierten Prozessen (Spreer et al. 2019 in Anlehnung an Imms et al. 2017, S. 19)

Imms et al. (2016) fanden heraus, dass viele Studien die Einteilung von Partizipation in zwei Komponenten gemeinsam hatten: „Attendance“, d.h. die reine Anwesenheit einer Person (teilnehmen an), von Spreer et al. (2019) übersetzt als „,Dabeisein“, welche gemessen wird als Häufigkeit der Teilnahme und/oder Reichweite oder Unterschiedlichkeit von Aktivitäten. Als zweite Komponente „Involvement“, was der Beteiligung und somit nach Spreer et al. (2019) dem „Eingebunden-sein“ (teilhaben an), entspricht. Das Konstrukt des „Eingebunden-seins“ enthält Erfahrungen, die während der „Anwesenheit“ gemacht werden, wie z.B. Engagement, Motivation, Ausdauer, soziale Verbindung und die Ebene des Affekts (beschäftigt sein in). Noch ist nicht endgültig geklärt wie beide Komponenten zusammenhängen (Spreer et al. 2019 nach Imms et al. 2016, 2017).

Ergänzt sind die beiden Komponenten durch die drei Elemente Präferenzen der Person, ihr Selbst-Verständnis und ihre Handlungskompetenz. Diese Bereiche sind einerseits wichtige Prädiktoren für die zukünftige Partizipation, andererseits werden sie durch bereits erlebte Partizipationserfahrungen beeinflusst (anerkannt werden) (Imms et al. 2016). Spreer et al. (2019, S. 216) beschreiben darauf Bezug nehmend den Einfluss der Präferenz der Kinder folgendermaßen: „Die Kinder wählen aus, an was sie teilnehmen werden [...], basierend auf früheren Beteiligungserfahrungen und den Erwartungen an eine zukünftige Teilnahme“. Dies bedeutet für den Gesundheitsbereich, dass die Partizipations- und Teilhabeinteressen eines Kindes, bzw. seiner Familie essentiell und richtungsweisend sind (Kraus de Camargo und Simon 2013): einerseits für das Ziel einer Behandlung oder Förderung, d.h. als „Kernzielgröße“ (Seidel 2015) bzw. dem bereits erwähntem „Endpunkt der Versorgung“ (Urschitz et al. 2016), andererseits, mit Bezug auf das genannte Stufenmodell, betreffend der (Mit-)Entscheidung über Behandlungsplan, Zielsetzung und Vorgehen. Hinter allen genannten partizipationszugehörigen Konstrukten stehen der Kontext, 
in welchem die Partizipation stattfindet, und die Umwelt. Zweites schließt zum Beispiel die Bereiche Verfügbarkeit, Zugang oder Finanzierung von möglichen Partizipationswünschen ein (Imms et al. 2016). Imms et al. (2017) heben hervor, dass diese Umgebungsbedingungen für die individuelle Partizipation äußerst bedeutsam sind, was sich auch in situationsabhängig unterschiedlicher Wahrnehmung der Partizipation äußert. Batorowicz et al. (2016) beschreiben die Umgebungsbedingen als „Kontext“, welcher notwendig ist, um Partizipation zu gestalten und auch zu beurteilen, da Menschen immer in Bezug zu ihrem Kontext handeln bzw. zum Handeln befähigt werden.

Zur Differenzierung von Dabei-Sein und Eingebunden-Sein stellt Sarimski (2015) in einer explorativen Studie aus dem inklusiven und integrativen Kita-Setting fest, dass Kinder mit Schwermehrfachbehinderungen zu einem Großteil der Zeit nur den Aspekt der reinen Anwesenheit erfüllen, soziale Teilhabe aber nur während knapp einem Drittel dieser Zeit stattfindet. Davon kann noch weniger als ,gelingende Kontakte“ zu anderen Kindern bezeichnet werden (Sarimski 2015, S. 149). Gerade auch für pädagogische Bildungs- und Lernprozesse ist der Aspekt des Engagements (beschäftigt sein in) bedeutsam und qualitativer Maßstab für die Frage, wie involviert ein Kind in Tätigkeiten, soziale Interaktionen oder Lernprozesse ist (Axelsson et al. 2013).

Die Familie der partizipationszugehörigen Konstrukte weist in ihrer Gesamtheit eine gute Anschlussfähigkeit an ein inklusives Bildungsverständnis auf, wie es bereits von Hollenweger (2015) in Bezug auf die ICF vorgenommen wurde: Partizipation heißt auch in Bildungs- und Lernprozessen, dass Kinder als selbstbestimmte Akteure und Akteurinnen angesehen werden und die Defizitorientierung durch Vermeidung von Eigenschaftszuweisungen überwunden wird. Demzufolge müssen Situationen, d. h. der Kontext, an die Kinder angepasst werden, um Lernen zu ermöglichen, denn „Lernen als Prozess findet in der Auseinandersetzung mit der Umwelt statt“" (Hollenweger 2015, S. 36).

Dies entspricht auch dem heutigen Verständnis und der Praxis, wie Kinder mit ihrer Entwicklung in frühpädagogischen Settings, sowohl inklusiv, als auch im Regelbereich, gesehen und gefördert werden: So ist bei Bildungs- und Entwicklungsplänen in der Frühpädagogik, die sich zwar an Entwicklungstheorien orientieren, trotzdem bedeutsam, ,in welcher Art und Weise Förder- und Bildungsabsichten auf ihre Bedürfnisse und Kompetenzen Bezug nehmen“" (Stamm 2010, S. 101). Die Umweltbedingungen, in diesem Fall Förder- und Bildungsabsichten, müssen also mit dem subjektiven Empfinden der Kinder in Einklang gebracht werden. Hierbei ist zweitrangig, ob die Kinder heterogen sind aufgrund unterschiedlicher Gesundheitszustände oder durch andere Themen, wie Herkunft, Sprache oder Entwicklungsstand.

\section{Fazit und Ausblick}

In dem Versuch verschiedene, für die Frühpädagogik und Frühförderung relevante Perspektiven auf Partizipation und Teilhabe zusammenzustellen, wird die Komplexität und Vielschichtigkeit der Thematik deutlich. Dies eröffnet einen Möglichkeits- 
raum, die eigene Haltung, Perspektive und das Verständnis von Partizipation und Teilhabe zu überdenken und disziplinübergreifend zu erweitern.

Im Zuge der Annahme und bestehender empirischer Erkenntnis, dass eine Verzahnung von frühkindlicher Bildung, Erziehung und Betreuung mit der Frühförderung ein zentral bedeutsamer Faktor für gelingende Inklusion ist (Seitz und Hamacher 2019), steigt die Bedeutsamkeit der Gestaltung von Kooperation und interdisziplinärer Zusammenarbeit mit der geteilten Leitidee zur Partizipation und Teilhabe von Kindern und ihren Familien. Dabei kommen Seitz und Hamacher (2019) zu der Schlussfolgerung, dass für diese inklusionsförderliche Kooperation die Orientierung an der ICF und dem bio-psycho-sozialen Modell hilfreich sein kann, wie auch im BTHG gefordert wird. Hierüber können einerseits Bedarfe erfasst werden und die Fachkräfte sensibilisiert werden, das kindliche Umfeld barrierearm zu gestalten und den Lebenskontext der Kinder und ihrer Familien konsequent einzubeziehen. Andererseits kann die ICF als Kommunikationsmittel gerade auch in der Kommunikation mit Leistungsträgern (Ämtern) Präzision und Klarheit schaffen. Jedoch sollte bei der Thematisierung von Partizipation und Teilhabe ein gemeinsames Verständnis in interdisziplinären Arbeitskontexten vorangestellt werden. Im Begleitforschungsprojekt zur inklusionsförderlichen Zusammenarbeit von Frühförderung und Kindertageseinrichtung wird dies deutlich: Hier zeigte sich ein divergentes Verständnis von Partizipation, welches zu Missverständnissen führen kann. Die Autorinnen kommen zu der Empfehlung, dass ein reflexiver Austausch für eine gemeinsame Bewusstseinsbildung notwendig und diesbezüglich die interprofessionelle Zusammenarbeit zu reflektieren sei (Seitz und Hamacher 2019).

Neben den zahlreichen Varietäten scheinen alle Begriffsdefinitionen und -nutzungen jedoch gemeinsam zu haben, dass die Stärkung der Rechte, die Selbstbestimmung und die individuellen Vorstellungen von Menschen elementar sind. Partizipation und Teilhabe bezeichnen somit immer einen Gegenpol zu Ausgrenzung, sei es auf Ebene von gesellschaftlichen Zugängen auf Makro- wie Mikroebene, bzgl. Entscheidungsmacht oder Verwirklichungschancen. Hierdurch wird u. a. die enge Verbindung von Partizipation und Inklusion sichtbar.

Die gemeinsame Nutzung der ICF kann helfen, Unterstützungsbedarfe sichtbar zu machen (Bedarfsermittlungsverfahren) und gemeinsame Zielperspektiven, z. B. in Bezug auf Diagnostik (Hollenweger 2012) sowie Bildungs- und Förderziele zu entwickeln. Wird pädagogische Diagnostik verstanden als Tätigkeit, durch die Voraussetzungen und Bedingungen planmäßiger Lehr- und Lernprozesse ermittelt, Lernprozesse analysiert und Lernergebnisse festgestellt werden (Ingenkamp und Lissmann 2005), so kann die ICF eine gute Grundstruktur bieten, um über gesundheitsbezogene Faktoren, die die Lernvoraussetzungen bei Kindern mit Beeinträchtigungen beeinflussen können. Ebenso können auch die Kontextfaktoren systematisch erfasst werden. In einem erweiterten Verständnis pädagogischer Diagnostik nach Breitenbach (2020) bedeutet dies, die Lern- und Lebenswelt eines Kindes und seiner Familie kennen zu lernen und die Welt aus Augen und Erleben des Kindes sehen können: Hierin liegt sicherlich die Stärke einer frühpädagogischen Fachperspektive: über eine Entwicklungsdiagnostik hinaus, die z.B. durch die Frühförderung geleistet werden kann, Partizipations- und Teilhabeprozesse zu analysieren. Die systematische Erhebung von Barrieren und Förderfaktoren innerhalb der ICF kann des Weiteren auch 
dazu genutzt werden, Strukturbedingungen (i.S.v. Qualitätsfeststellungsverfahren, Fried 2011) systematisch zu erfassen. Die Einrichtung kann sich somit bzgl. der Ermöglichung von Partizipation und Teilhabe, sowie dementsprechend von Inklusion, reflektieren.

Partizipation und Teilhabe weisen in Frühpädagogik und Frühförderung viele Gemeinsamkeiten auf, die durch eine gemeinsame Sprache der ICF zu einem abgestimmten, interdisziplinären Handeln führen können.

Funding This Article is funded by the Open Access Publication Fund of South Westphalia University of Applied Sciences.“

Funding Open Access funding enabled and organized by Projekt DEAL.

Open Access Dieser Artikel wird unter der Creative Commons Namensnennung 4.0 International Lizenz veröffentlicht, welche die Nutzung, Vervielfältigung, Bearbeitung, Verbreitung und Wiedergabe in jeglichem Medium und Format erlaubt, sofern Sie den/die ursprünglichen Autor(en) und die Quelle ordnungsgemäß nennen, einen Link zur Creative Commons Lizenz beifügen und angeben, ob Änderungen vorgenommen wurden.

Die in diesem Artikel enthaltenen Bilder und sonstiges Drittmaterial unterliegen ebenfalls der genannten Creative Commons Lizenz, sofern sich aus der Abbildungslegende nichts anderes ergibt. Sofern das betreffende Material nicht unter der genannten Creative Commons Lizenz steht und die betreffende Handlung nicht nach gesetzlichen Vorschriften erlaubt ist, ist für die oben aufgeführten Weiterverwendungen des Materials die Einwilligung des jeweiligen Rechteinhabers einzuholen.

Weitere Details zur Lizenz entnehmen Sie bitte der Lizenzinformation auf http://creativecommons.org/ licenses/by/4.0/deed.de.

\section{Literatur}

Axelsson, A. K., Granlund, M., \& Wilder, J. (2013). Engagement in family activities: a quantitative, comparative study of children with profound intellectual and multiple disabilities and children with typical development. Child Care Health and Development, 39(4), 523-534. https://doi.org/10.1111/cch. 12044.

BAR - Bundesarbeitsgemeinschaft für Rehabilitation (2016). Nachhaltigkeit von Leistungen zur Rehabilitation und Teilhabe. Handlungsempfehlungen. Frankfurt a.M.: BAR.

Batorowicz, B., King, G., Mishra, L., \& Missiuna, C. (2016). An integrated model of social environment and social context for pediatric rehabilitation. Disability and Rehabilitation, 38(12), 1204-1215. https://doi.org/10.3109/09638288.2015.1076070.

Bertelsmann Stiftung (2019). Ländermonitor frühkindliche Bildungssysteme: Kinder mit Eingliederungshilfe in Kitas nach Betreuungsform. https://www.laendermonitor.de/de/vergleich-bundeslaenderdaten/kinder-und-eltern/inklusion/kinder-mit-eingliederungshilfe-in-kitas-nach-betreuungsform. Zugegriffen: 1. Apr. 2021.

BMAS - Bundesministerium für Arbeit und Soziales (2016). Mehr möglich machen - weniger behindern. Das neue Bundesteilhabegesetz. https://www.bmas.de/DE/Soziales/Teilhabe-und-Inklusion/ Rehabilitation-und-Teilhabe/bundesteilhabegesetz.html. Zugegriffen: 5. Okt. 2020.

Breitenbach, E. (2020). Diagnostik. Eine Einführung. Wiesbaden: Springer VS.

Fried, L. (2011). Frühpädagogische Diagnostik. In K.-P. Horn, H. Kemnitz, W. Marotzki \& U. Sandfuchs (Hrsg.), Klinkhardt-Lexikon Erziehungswissenschaft (S. 437-439). Bad Heilbrunn: Klinkhardt, UTB.

Hansen, R., Knauer, R., \& Sturzenhecker, B. (2011). Partizipation in Kindertageseinrichtungen. So gelingt Demokratiebildung mit Kindern. Weimar, Berlin: Verlag das Netz.

Heimlich, U., \& Ueffing, C. (2018). Leitfaden für inklusive Kindertageseinrichtungen. Bestandsaufnahme und Entwicklung. München: Deutsches Jugendinstitut e. V.. WiFF-Expertise

Hirschberg, M. (2010). Partizipation - ein Querschnittsanliegen der UN-Behindertenrechtskonvention. Positionen der Monitoring-Stelle, Bd. 3/2010. Berlin: Deutsches Institut für Menschenrechte. 
Hollenweger, J. (2012). Partizipative Erkundungen. Diagnostik als Voraussetzung für Bildungsprozesse. Pädagogische Impulse, 3, 10-20.

Hollenweger, J. (2015). Anwendung der ICF im Kontext von Lernen und Lernstörungen. Lernen und Lernstörungen, 4(1), 31-41.

Imms, C., Adair, B., Keen, D., Ullenhag, A., Rosenbaum, P., \& Granlund, M. (2016). 'Participation': a systematic review of language, definitions, and constructs used in intervention research with children with disabilities. Developmental Medicine and Child Neurology, 58(1), 29-38. https://doi.org/ $10.1111 /$ dmen. 12932.

Imms, C., Granlund, M., Wilson, P.H., Steenbergen, B., Rosenbaum, P.L., \& Gordon, A. M. (2017). Participation, both a means and an end. A conceptual analysis of processes and outcomes in childhood disability. Developmental Medicine and Child Neurology, 59(1), 16-25. https://doi.org/10.1111/dmcn. 13237.

Ingenkamp, K., \& Lissmann, U. (2005). Lehrbuch der pädagogischen Diagnostik (5. Aufl.). Weinheim: Beltz.

von Kardorff, E. (2014). Partizipation im aktuellen gesellschaftlichen Diskurs - Anmerkungen zur Vielfalt eines Konzepts und seiner Rolle in der Sozialarbeit. Archiv für Wissenschaft und Praxis der sozialen Arbeit, 2, 4-15.

Knauer, R. (2012). Eine Verfassung für Kinderrechte? Beteiligung als Voraussetzung für Bildungsprozesse in Kindertageseinrichtungen. In S. Penka \& R. Fehrenbacher (Hrsg.), Kinderrechte umgesetzt. Grundlagen, Reflexion und Praxis (S. 65-74). Freiburg: Lambertus.

Kraus de Camargo, O., \& Simon, L. (2013). Die ICF-CY in der Praxis. Bern: Huber.

Kreuzer, M., \& Ytterhus, B. (2013). „Dabeisein ist nicht alles“ - Inklusion und Zusammenleben im Kindergarten (3. Aufl.). München, Basel: Ernst Reinhardt.

Kronauer, M. (2017). Exklusion und soziale Teilhabe in Deutschland und Perspektiven für eine solidarische und demokratische Entwicklung. BAG Wohnungslosenhilfe. Berlin. https://www.bagw.de/ media/doc/TGD_17_BUTA-Vortrag-Kronauer.pdf. Zugegriffen: 14. Okt. 2020.

Liebel, M. (2013). Kinder und Gerechtigkeit. Über Kinderrechte neu nachdenken. Weinheim, Basel: Beltz Juventa.

MSW - Ministerium für Schule und Bildung des Landes Nordrhein-Westfalen (2018). Bildungsgrundsätze. Mehr Chancen durch Bildung von Anfang an; Grundsätze zur Bildungsförderung für Kinder von 0 bis 10 Jahren in Kindertagesbetreuung und Schulen im Primarbereich in Nordrhein-Westfalen (2. Aufl.). Freiburg, Basel, Wien: Herder.

Nentwig-Gesemann, I., \& Maywald, J. (2020). Editorial Partizipation und Kinderrechte. Frühe Bildung, 9(1), 1-2. https://doi.org/10.1026/2191-9186/a000460.

Peucker, C., Pluto, L., \& van Santen, E. (2017). Situation und Perspektiven von Kindertageseinrichtungen. Empirische Befunde (1. Aufl.). Weinheim, Basel: Beltz Juventa.

Prengel, A. (2016). Bildungsteilhabe und Partizipation in Kindergartentageseinrichtungen. München: Deutsches Jugendinstitut e. V.. WIFF-Expertise

Pretis, M. (2016). ICF-basiertes Arbeiten in der Frühförderung. München, Basel: Ernst Reinhardt.

Sarimski, K. (2015). Dabeisein ist nicht alles - oder doch?! Frühförderung interdisziplinär, 34, 141-151. https://doi.org/10.2378/fi2015.art18d.

Seidel, A. (2015). Theoretische und praktische Aspekte für die Anwendung der ICF-CY in der Neuropädiatrie. Neuropädiatrie in Klinik und Praxis, 14(2), 56-63.

Seitz, S., \& Hamacher, C. (2019). Abschlussbericht des Modellprojektes „Teilhabechancen für Kinder verbessern - Kooperation von Frühförderstellen und Tageseinrichtungen für Kinder stärken ". Caritasverband des Erzbistums Paderborn

Simon, L., \& Philippi, H. (2019). Einführung in das Arbeiten mit der ICF in Frühförderung und Sozialpädiatrie. In B. Gebhard, S. Möller-Dreischer, A. Seidel \& A. Sohns (Hrsg.), Frühförderung wirkt von Anfang an (S. 193-200). Stuttgart: Kohlhammer.

Spreer, M., Fink, A., \& Gebhard, B. (2019). Stichwort: Partizipation. Frühförderung Interdisziplinär, 38(4), 214-217. https://doi.org/10.2378/fi2019.art27d.

Stamm, M. (2010). Frühkindliche Bildung, Betreuung und Erziehung. Stuttgart: UTB.

Thon, C., \& Mai, M. (2017). Inklusion oder Teilhabe nach Plan? Über die Pädagogisierung eines politischen Anliegens im Kontext frühkindlicher Bildung. In I. Miethe, A. Tervooren \& N. Ricken (Hrsg.), Bildung und Teilhabe. Zwischen Inklusionsforderung und Exklusionsbedrohung (S. 259-278). Wiesbaden: Springer VS.

Urschitz, M., Gebhard, B., Philippi, H., \& DeBock, F. (2016). Partizipation und Bildung als Endpunkte in der pädiatrischen Versorgungsforschung. Zeitschrift Kinder- und Jugendmedizin, 16(3), 206-217. 
Weiß, H. (2019). Teilhabe im Kontext der Frühförderung. Frühförderung Interdisziplinär, 38(4), 191-206. https://doi.org/10.2378/fi2019.art25d.

WHO - World Health Organisation (2005). ICF - Internationale Klassifikation der Funktionsfähigkeit, Behinderung und Gesundheit. Köln: Bundesinstitut für Arzneimittel und Medizinprodukte (BfArM).

WHO - World Health Organisation (2011). ICF-CY. Internationale Klassifikation der Funktionsfähigkeit, Behinderung und Gesundheit bei Kindern und Jugendlichen (2. Aufl.). Bern: Hogrefe. Übersetzt und herausgegeben durch J. Hollenweger \& O. Kraus de Camargo (2017)

Wiedebusch, S., Lohmann, A., Tasche, H., Thye, M., \& Hensen, G. (2015). Inklusion von Kindern mit Beeinträchtigungen im Spiegel pädagogischer Konzeptionen von Kindertageseinrichtungen. Frühe Bildung, 4(4), 203-210. https://doi.org/10.1026/2191-9186/a000206.

Wright, M. T., Block, M., \& von Unger, H. (2007). Stufen der Partizipation in der Gesundheitsförderung: Ein Modell zur Beurteilung von Beteiligung. Infodienst für Gesundheitsförderung, 3, 4-5.

Wright, M. T., Block, M., \& von Unger, H. (2010). Partizipation der Zielgruppe in der Gesundheitsförderung und Prävention. In M. T. Wright (Hrsg.), Partizipative Qualitätsentwicklung in der Prävention und Gesundheitsförderung (S. 35-52). Bern: Huber. 